\title{
Characteristic frequency of muscle response to electrical stimulation in normal and hypokalemic rats
}

\author{
Member \\ Atsuo Nuruki \\ (Kagoshima University) \\ Non-Member \\ Ryoji Nagaoka \\ (Kagoshima University) \\ Non-Member \\ Gang Wang \\ (Kagoshima University) \\ Member \\ Kazutomo Yunokuchi \\ (Kagoshima University)
}

\begin{abstract}
This paper examines frequency characteristics of muscle, which show differences between slow and fast twitch fiber type muscle in normal and hypokalemic rats. The characteristics of muscle stimulating frequencies and EMG amplitude was studied by using control theory. The EMG was recorded from soleus (slow twitch fiber type) and extensor digitorum longus (fast twitch fiber type) in vivo. It was found that muscle showed different frequency response, and that under hypokalemia each muscle showed poor response. Furthermore, in response to electric stimulation to achieve an equivalent level of response, it was found that muscle needed higher electric intensity than nerve-muscle and muscle showed remarkable decay under hypokalemia. These results suggest that the main disease origin under hypokalemia can be ascribed to muscle condition, and that it is most efficient to stimulate nerves in order to control muscle.
\end{abstract}

keywords: frequency response, hypokalemia, muscle, control theory, break point frequency

\section{Introduction}

The principles of the central and peripheral control mechanisms of muscle are becoming better understood as a result of numerous medical and neurophysiological studies. Although many studies have been carried out relating to nerve-muscle activity in bioengineering, it is difficult to match the experimental and logical results to human application, because our motor system is regulated by a great diversity of internal and external demands and constraints.

For a subject with tetraplegia, many research groups have shown that partial restoring of reaching and grasping, which are among the main objectives of rehabilitation to paralyzed limbs, can be achieved through neural prosthetic systems that use functional electrical stimulation (FES) to activate the dormant muscles [1-3]. FES systems use controlled electrical currents to activate muscles that have lost their connection to the spinal cord and higher level control centers, but still have their peripheral nerve supplies intact. However, FES input methods are not able to finely control the muscles of paralyzed patients despite much research. In this paper, we investigate the frequency response of the muscle, with particular application to disease. In FES systems, altering the amplitude, pulse width and frequency for stimulation are used to control muscle contraction. Particularly, altering the frequency of stimulation seems to resemble the input pattern of real nervous signal which is variation of firing rate. However, few studies on frequency of stimulation have been carried out [4-6]. Furthermore, the reasons why muscle fatigues far more rapidly when artificially stimulated (using modulation of amplitude and pulse width) than when excited by the central nervous system are not entirely understood. So, much work remains to achieve better control of paralyzed muscles, as seen in normal movement.

From this background, the author studied that the nerve-muscle response to altering frequency of stimulation and showed the nerve-muscle have individual characteristics by the type of fiber, and the degree of nerve-muscle disease and fatigue [7] using control theory. Applying control theory to motor system was useful but at the bigging. However, muscle contraction is not only controlled by input into nervous system and the muscle itself has properties enabling modification of its input and contraction response. From the results of nerve-muscle response we modeled the nervemuscle and simulated frequency response from the nervemuscle system which can be expressed as a closed loop transfer function [8]. This nerve-muscle model consists of following transfer functions; nerve, end-plate, proprioceptor and muscle. Then, ensuring research should strived to identify individual peripheral nerve, end-plate, proprioceptor and muscle response. The frequency response of muscle must be investigated from a standpoint of control theory. Clarification of this information is necessary for the accurate planning of goal directed movements, for locomotor tasks and for postural adjustments in rehabilitation of neuromuscular disorders as FES. 
Additionally, the input-output properties of the muscle seems to be as a diagnosis of neuromuscular dysfunction.

In this paper, we examines frequency response of muscle, which show differences between slow and fast twitch fiber type in normal and hypokalemic rats which is a model relevant to hypokalemic periodic paralysis. It is also important to obtain the differences of property in frequency response between normal and hypokalemic muslce in order to diagnose the degree and part of disease.

\section{Materials and Methods}

\subsection{Animal preparation and muscle model}

Rats were divided into 2 groups consisting of control and hypokalemia. The control group consisted of Male wistar strain rats. Control group rats had free access to food and water and were housed in a climate-controlled room at $25^{\circ} \mathrm{C}$ and $60 \%$ humidity, starting at an age of four to five weeks-old, for five weeks. For the disease state, we chose hypokalemia as a chronic nerve-muscle disease model. These rats suffered from hypokalemic periodic paralysis.

Hypokalemic rats were created by feeding food and water which did not contain potassium $\left(\mathrm{K}^{+}\right)$to male Wistar strain rats starting at an age of four to five weeks-old, for five weeks, in conditions otherwise identical to the control rats.

We chose two muscle fiber type models consisting of the Soleus; (SOL), a slow twitch fiber type model; and the Extensor Digitorum Longus; (EDL), a fast twitch fiber type model.

\subsection{Experimental procedure}

In both groups, rats 9 to 11 weeks-old weighing between 200 and $250 \mathrm{~g}$ were used. Rats were anesthetized with intraperitoneal injection of somnopentil (containing $64.8 \mathrm{mg} / \mathrm{ml}$ of pentobarbital sodium. Pitaman-Moore, Inc., U.S.A.) at a dose of $6.25 \mathrm{mg} / 100 \mathrm{~g}$ body weight. The hind limb was fixed horizontally by firmly clamping the ankle with clips onto the table.

\subsection{Measurement of frequency response \\ 1) Muscle response}

The SOL and EDL were exposed by skin incision. Nervous function remained intact and muscle blood supply was not disrupted. The bipolar stimulation electrode was placed over the muscle surface, motor point. The system of muscle response was same condition except for a position of stimulation electrode.

\subsection{Applying control theory to nerve-muscle}

In general, a sine waveform in control theory is used for input in order to estimate the frequency response of a device. However, nerves and muscles do not respond well to sine wave input, so we used a rectangular input waveform. Fig. $\mathrm{lb}$ is schematic of the concept of this experiment based on control theory. On the left of Fig. la, T is pulse interval, $A$ is pulse amplitude and $\mathrm{Tw}$ is pulse durations which is determined by rat's strength-duration curve muscle [8]. For output, amplitude of evoked potentials were used.

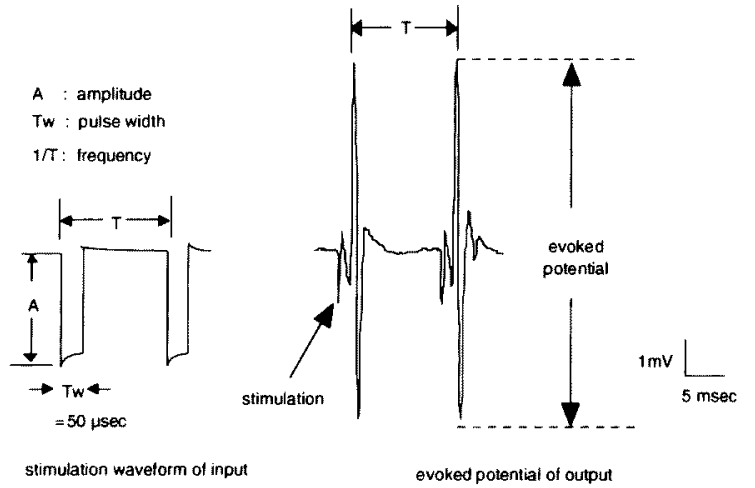

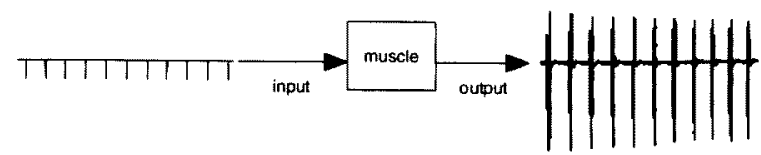

a series of evoked potential

Fig. 1. (a) Measurement of frequency response of muscle. (b) Schematic of the concept of this experiment based on control theory. In general, a sine waveform is used for input in control theory. However, the muscle is unable to respond well to the input sine waveform, so we chose a rectangular input waveform. In the left figure of (a), $\mathrm{T}$ is pulse intervals, $\mathrm{A}$ is pulse amplitude and $\mathrm{Tw}$ is pulse duration which is determined by rat's strength-duration curve of muscle[8]. The electric stimulation was varied from a frequency of $0.5 \mathrm{~Hz}$ to $200 \mathrm{~Hz}$ by the changing of duration T. One trial consisted of a series of 11 stimulations. Measurements were taken from the second response to the last response. The first response was used as a control, with the same amplitude evoked for all frequencies. The right figure of (a) shows actual evoked potential of output measured peak to peak. For each trials frequency control theory (b) was applied to the association between stimulation input and potential voltage represented in decibels (dB). 


\subsection{Electric stimulation}

Threshold stimulation voltage was determined by first increasing the voltage until the EMG was obtained. The frequency dependency tests were carried out with the electric stimulation varied from a frequency of 0.5 to $200 \mathrm{~Hz}$ (NEC Sanei Co., Ltd. JAPAN, Type 3F 46) at $15-20 \%$ above threshold voltage. One trial consisted of a series of 11 stimulations. Measurements were taken from the second response to the last response. The first response was used as a control, with the same amplitude evoked for all frequencies. Each frequency test included two minute rest intervals in order to recover from fatigue. Each experiment was repeated not less than 10 times to verify results.

\subsection{EMG signal detection}

The electromyogram (EMG) was recorded from the SOL and EDL using two silver-silver chloride electrodes, $0.5 \mathrm{~mm}$ diameter. Surface recording electrodes separated by $5 \mathrm{~mm}$ were placed parallel to the muscle fiber. Bipolar recordings were made with the reference electrode around the limb tendon.

\subsection{EMG signal processing and data analysis}

EMG signals were sampled at a rate of $10 \mathrm{kHz}$ and amplified (NIHONSANEI Polygraph 360 system 365 I-B, JAPAN) using band-pass filter between 0 and $1 \mathrm{kHz}$. The amplified EMG signals were then digitized with an analog to digital converter (MacLab v.8s) connected to a Macintosh PowerBook computer (1400cs/133). Analysis of recorded signals were performed using Maclab chart v3.5.2/s analysis software. Data are presented as means, with data error calculated to be $\pm 2.5 \%$ (not shown in the figures for the sake of clarity). The stimulation input voltage and the evoked potential are represented in decibels $(\mathrm{dB})$ for comparison purposes.

\section{Results}

Frequency response of muscles

The results of our experiments are summarized in Table 1 , showing derived relative gain at $0.5 \mathrm{~Hz}$, different gain between $0.5 \mathrm{~Hz}$ and $20 \mathrm{~Hz}(\Delta \mathrm{G} 0.5-20)$, and different gain between $20 \mathrm{~Hz}$ and $100 \mathrm{~Hz}(\Delta \mathrm{G} 20-100)$, for control and hypokalemic SOL and EDL of muscle response.

\subsection{Muscle responses}

Fig. 2 presents the different frequency response curves of the muscle for normal SOL and EDL. Gain of SOL and EDL were at about $-53.60 \mathrm{~dB}$ and $-56.31 \mathrm{~dB}$ respectively, at $0.5 \mathrm{~Hz}$. Frequency response curves of the nerve-muscle for SOL and EDL in the hypokalemic state are shown in Fig. 3. SOL and EDL were at about $-59.22 \mathrm{~dB}$ and $-55.40 \mathrm{~dB}$ respectively, at $0.5 \mathrm{~Hz}$. Under low frequency stimulation, $\triangle$ G0.5-20 of SOL and EDL under normal conditions registered $-1.62 \mathrm{~dB}$ and $-4.10 \mathrm{~dB}$, respectively and $\Delta \mathrm{G} 0.5-20$ of SOL and EDL under hypokalemic conditions registered $3.10 \mathrm{~dB}$ and $-6.14 \mathrm{~dB}$, respectively. Under high frequency stimulation, $\triangle \mathrm{G} 20-100$ of SOL and EDL under normal conditions registered $-10.11 \mathrm{~dB}$ and $-5.95 \mathrm{~dB}$, respectively and $\triangle \mathrm{G} 20-100$ of SOL and EDL under hypokalemic conditions registered $-2.25 \mathrm{~dB}$ and $-12.00 \mathrm{~dB}$, respectively.

\section{Discussion}

The purpose of this work was to obtain a useful model of muscle control, to aid with diagnosis of muscle disease, and represents a promising approach to the stimulation profiles in FES. Our approach was to apply the principles of control theory to muscle evoked potential by varying the frequency of electric stimulation. In this study there are two approaches to muscle properties: bioengineering and neuromuscular disorders. Because we want to control actual muscle contraction for applications such as FES, from a

Table 1. Frequency response of muscle in SOL and EDL under normal and hypokalemic condition.

\begin{tabular}{|c|c|c|c|c|}
\hline & G0.5 (dB) & $\Delta \mathrm{G} 0.5-20(\mathrm{~dB})$ & $\Delta \mathrm{G} 20-100(\mathrm{~dB})$ & $n$ \\
\hline Normal (SOL) & $-53.60 \pm 3.24$ & $-1.62 \pm 0.55$ & $-10.11 \pm 2.76$ & 6 \\
\hline Normal (EDL) & $-56.31 \pm 3.13$ & $-4.10 \pm 1.06$ & $-5.95 \pm 1.28$ & 5 \\
\hline Hypokalcmic (SOL) & $-59.22 \pm 2.62$ & $-3.94 \pm 1.59$ & $-1.73 \pm 1.10$ & 7 \\
\hline Hypokalemic (EDL) & $-55.40 \pm 2.180$ & $-6.14 \pm 2.21$ & $-12.00 \pm 2.61$ & 5 \\
\hline
\end{tabular}




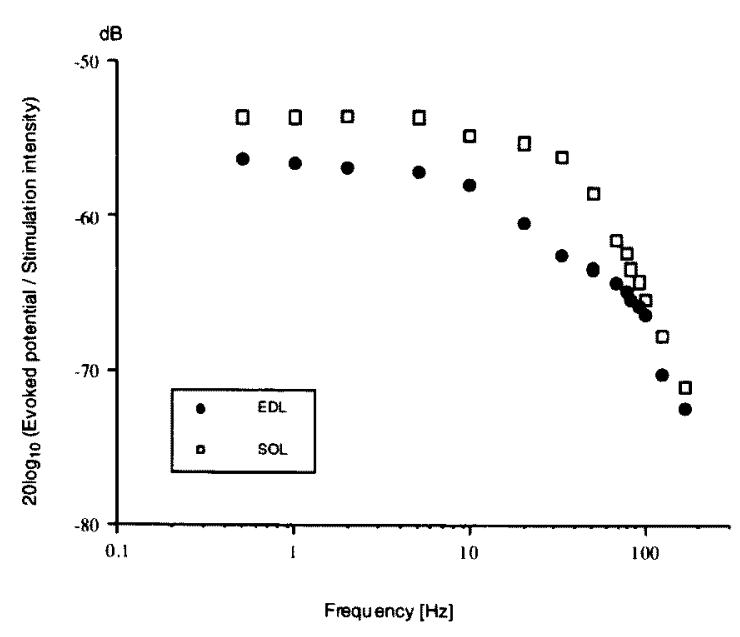

Fig. 2 Muscle frequency response curves of normal SOL and EDL.

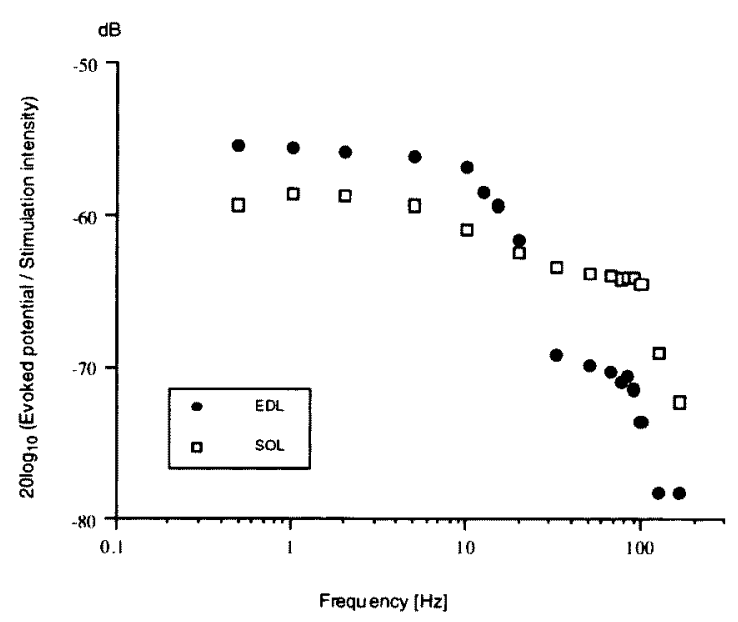

Fig. 3. Muscle frequency response curves of hypokalemic SOL and EDL.

standpoint of bioengineering it is important to examine the relationship between the frequency of electric stimulation and the muscle force. In FES, the muscle strength is commonly controlled by varying both recruitment and temporal summation of the muscle fibers activated through altering the stimulation pulse width (PW) [9] or the stimulation frequency. Basically, researchers using FES want to know the variation of muscle force response to the frequency of stimulation. In contrast, the majority of physiologists want to know chemical and electrophysiological data to clarify the mechanisms of nerve-muscle activity. We have used elements of both fields in this study to obtain a more comprehensive understanding of neuromuscular properties. In our previous research, we studied that the nerve-muscle response to altering frequency of stimulation and showed the nerve-muscle have individual characteristics by the type of fiber, and the degree of nervemuscle disease and fatigue [7] using control theory. From these results, we concluded that desease condition with hypokalimia ascrive to muscle, but not clear. Then, from the results of nerve-muscle response we modeld the nervemuscle and simulated frequency response from the nervemuscle system which can be expressed as a closed loop transfer function [8], consists of follwing transfer function; nerve, end-plate, proprioceptor and muscle. Therefore, ensuring research should strived to identify individual peripheral nerve, end-plate, proprioceptor and muscle response. From these background, we examined the relationship between the evoked potential and the frequency of stimulation, using both slow and fast twitch muscle fibers, in normal and hypokalemic muscle.

Both muscle frequency responses were investigated. In every case, muscle frequency responses were remarkably worse than nerve-muscle frequency responses of [7](see Fig. 2 and Fig. 3). Response of muscle to both high and low frequency stimulation was worse than nerve-muscle response. These results indicate that in order to control muscle it is more efficient to stimulate nerves than muscles. However, under hypokalemia, evoked potential for both high and low frequency stimulation to muscle showed similar tendencies to that of nerve-muscle. Therefore, it is suggested that the main origin of disease under hypokalemia is ascribable to muscle condition. From a biochemical standpoint, this idea demonstrated that hypokalemia occurs in muscle where potassium levels are about three times lower than normal [10]. That is, in hypokalemia there is a failure of muscle excitation or the excitation-contraction coupling is interrupted, relating to possible inactivation of the sodium-potassium pump due to the low concentration of extracellular potassium.

In this study, we investigated muscle response using principles of control theory. Our experiments demonstrated the controlling factors of nerve-muscle condition, and showed that a neuromuscular control system using electric stimulation is more efficient when applied to nerve than muscle. Then, we were able to estimate several characteristics of muscle type and the origin and degree of hypokalemia that results in muscle paralysis. Furthermore, these results of muscle response in this paper seems to be help to make individual transfer function, nerve, end-plate and muscle.

(Manuscript received Jan.15, 2002, revised April 8, 2002) 


\section{Reference}

(1) R.J. Jaeger, G. Yarkony, R. Smith: "Standing the spinal cord injured patient by electrical stimulation: refinement of a protocol for clinical use", IEEE Trans. on Biomed. Eng., 36, 720 728 (1989)

(2) L.J. Miller, P.H. Peckham, M.W. Keith: "Elbow extension in the C5 quadriplegic using functional neuromuscular stimulation", IEEE Trans. on Biomed. Eng., 37, 771 780 (1990)

(3) C. Veraart, W.M. Grill, J.T. Mortimer: "Selective control of muscle activation with a multipolar nerve cuff electrode", IEEE Trans. on Biomed. Eng., 40, 640 653 (1993)

(4) H.J. Chizek, N. Lan, L. Streeter Palmieri, P.E. Crago: "Feedback control of electrically stimulated muscle using simultaneous pulse width and stimulus period modulation", IEEE Trans. on Biomed. Eng., 38, 1224 1234 (1991)

(5) N. Lan, P.E. Crago, H.J. Chizeck: "Feedback control methods for task regulation by electrical stimulation of muscles", IEEE Trans. on Biomed. Eng., 38, 1213 1223 (1991)

(6) T. Watanabe, K. Morita, H. Murakami, N. Hoshimiya, Y. Handa: "Measurement of stimulus frequency-force characteristics for FES control method including differences between fast and slow muscle properties", lyoudenshi., 34, 47 51 (1996)

(7) A. Nuruki, S. Uchida, K. Yunokuchi and R. Nagaoka: "Frequency response of evoked potential in normal and diseased nerve muscle", IEEE Eng. Med. and Biol., 18, No. 6, 27 -32 (1999)

(8) A. Nuruki, K. Tanaka, G. Wang and Yunokuchi : "Modeling and simulation of frequency response of nerve-muscle", IEICE Trans. on COMMUN., E85-D, 199 202 (2001)

(9) P.E. Crago, P.H. Peckham, G.B. Thrope: "Modulation of muscle force by recruitment during intramuscular stimulation", IEEE Truns. on Biomed. Eng., 27, 679 684 (1980)

(10) N. Akaike: "Cation concentration change in rat skeletal muscles associated with potassium deficiency and denervation", Jpn $J$. Physiol. , 19, 420 438 (1969)
Atsuo Nuruki (Member) received Dr. Engineering degree from University of Kagoshima in 2001. Currently, he is a Research Associate of Department of Bioengineering. His research interests include identification and modeling of muscle control system consists of the brain, spinal cord, peripheral nerve, end-plate and muscle. $\mathrm{He}$ is a member of IEEE, Institute of Electronics, Information and Communication Engineers of Japan and the Japan society of Clinical neurophysiology and so on.

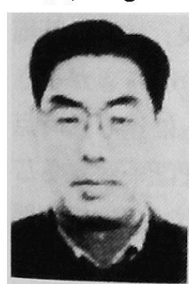

1 in Yamaguchi, Japan. He received the M.A. degree in health and fitness from Juntendo University, Tokyo, in 1976, and the Ph.D. degree in neuro-muscular physiology from Hiroshima University, Hiroshima, in 1990. He is currently an associate professor at the department of health education of Kagoshima University. His research interests include muscle $\mathrm{Na}^{+}-\mathrm{K}^{+}$pump activity in hypokalemia.
Gang Wang (Non-member) received M.S. degrees from University of Kagoshima, Japan. He received the Ph. D. degree from the department of medicine, University of Kagoshima, Japan, in 1993. He worked at the Institute of physical and chemical Research as a frontier researcher from 1994 to 1995, at the department of physiology, faculty of medicine as a research associate from 1996 to 1998 . He has been an associate professor with department of bioengineering, University of Kagoshima since 1999. His research interest includes brain science, higher functions of central nervous system. He is a member of Japan Neuroscience society, Japan society of medical engineering and so on.

Kazutomo Yunokuchi (Member) worked at Francis Bitter Magnet

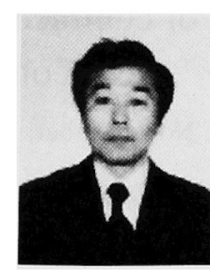
Laboratory in MIT as a visiting researcher from 1989 to 1991. He received Dr. Engineering degree from University of Tokyo in 1993. He is a professor of Department of Bioengineering, Kagoshima University. His current interest is in the area of biomagnetism. He is a member of IEEE, Institute of Electronics, Information and Communication Engineers of Japan and the Japan society of medical engineering. 